\title{
How many inpatient paediatric units do we need?
}

\author{
Brent Taylor
}

District general hospital paediatrics in this country is not keeping up with changes in the pattern of diseases, the need for specialisation, advances in secondary community and primary health care, the demands of training, the associated need for innovative service developments and research. Most present paediatric units are too small to provide safe and cost effective care; most do not meet the required standards for care or training. Adjacent units should combine to form larger comprehensive specialist children's services, incorporating specialist community care, increased day care facilities, and with fewer but larger units providing 24 hour care.

Paediatrics is changing. The average duration of hospital stay for medical problems continues to fall, suggesting that most children are less seriously ill than hitherto when admitted. Primary care continues to develop. General practitioners are doing more and dealing competently with many problems previously referred. Other community child health services are also doing more, including work previously done in hospital paediatric units. The interface between primary and secondary care is constantly changing and is often blurred, with similar clinical problems dealt with by general practitioners in some areas and specialist paediatricians in others. General practitioners provide most child health medical care but need back-up and support, especially for rare or complex problems. The quality of back-up and support tends to vary; the Audit Commission reported ${ }^{1}$ a more than fourfold variation in admission rates for asthma suggesting that many paediatric units were admitting unnecessarily. Overall admission rates for most conditions are apparently not falling and may possibly be rising; it is likely this is not real, but reflects changes in definitions (consultant episodes replacing hospital discharges) and better recording of data with improved information systems, associated with the need to keep better records in the more market oriented NHS.

The pattern of disease is changing. Many children are surviving previously fatal conditions such as cancer or cystic fibrosis; new patterns of disease are appearing (for example, changing types of cerebral palsy in association with the survival of very preterm babies); and certain chronic conditions, for example, diabetes, severe asthma and eczema, have shown a marked increase in prevalence over recent decades. When children with these conditions require inpatient treatment, they are likely to require high dependency care or intensive care, not often available and often not optimally provided in small units. Most care for these and other conditions, however, is not critical and should be provided on an outpatient or ambulatory basis, close to where the families live to ensure minimal disruption to relationships and schooling.

\section{Present problems}

\section{(1) QUALITY OF CARE}

Most present paediatric inpatient units provide a 'Jack of all trades' service. There is general acceptance that the occasional surgical operator is dangerous; neonatal surgical units are recommended to undertake at least 100 major operations in a year. A recent British Paediatric Association (BPA) report after the Confidential Enquiry into Peri-Operative Deaths recommended centralisation of all surgery in children under 3 years of age. ${ }^{2}$ Similar arguments can, and perhaps should, apply to paediatric medical practice. The Audit Commission recommends a minimum of 500 intensive care days per year to qualify for level 2 neonatal unit status. ${ }^{1}$ Conditions requiring high dependency or intensive care, like meningococcal sepsis, do better in specialist centres. Many difficult cases are referred on from smaller to specialist units; this should happen more. Recently trained and initially competent staff, medical, nursing and others, become rapidly deskilled and less competent without continuing experience. The maintenance of competence to deal with difficult or unusual cases needs adequate throughput. This is a quality issue and should be incorporated into contracts if children are to receive optimal care.

\section{(2) OPPORTUNITY TO SPECIALISE}

Jack of all trades consultants, on-call in most paediatric units one night in three or one night in four and often called in, may have a special interest in diabetes, or difficult asthma, and/or take the lead in the neonatal unit. However, low case numbers and low throughput mean there is limited opportunity to feel more than a dabbler, rarely an innovator. Specialist units in tertiary centres should be promoting tertiary services (rather than just the tertiary centre), by actively pursuing 'shared care' provided close to where the child lives, in a community 
setting whenever possible. Individual adverse experiences, when shared care is tried before being properly organised, often result in care returning to the (usually distant) tertiary centre, to the detriment of children and families in terms of travel time and opportunity costs, and to the detriment of the tertiary centre in terms of submersion under routine clinical work at the expense of time for service development and research. Trust needs to be developed between tertiary centres and secondary/primary services, with closer cooperation, agreed protocols, free and accessible communication to discuss issues/ problems, and a commitment on all sides to local provision for most aspects of care/ monitoring of the condition. Tertiary centres should limit their clinical activity to initial assessment and unusual complications, concentrating on development and shared care (which is not just undertaking peripheral clinics). Shared care can be difficult; the nominated 'specialist' district general hospital consultant may go on holiday or be away or distracted with other clinical matters when problems arise; managerial, child advocate/counsellor, trainer/teaching responsibilities, and increasing involvement in audit and continuing medical education can further erode the concentration of a district general hospital paediatrician perpetuating the 'Jack of all trades, master of none' status. A larger, critical mass of consultants in a larger paediatric unit would allow individuals to concentrate on fewer specialty clinical areas, while maintaining some 'general' clinical activity and rotation of managerial and other responsibilities. Greater depth of experience would increase confidence and competence for the nominated consultant and deputy (who would provide cover and local support).

\section{(3) ADVERSE EFFECT ON DEVELOPMENT OF SPECIALIST COMMUNITY CHILD HEALTH}

The current accent on inpatient facilities, and the perceived need to maintain current arrangements, has resulted in consultant community paediatricians and other community staff contributing to paediatric on-call work. In many units the involved consultants provide a full oncall commitment including cover for neonatal intensive care. Most do ward rounds and hospital outpatient sessions, often as many as their full time hospital paediatric colleagues. Hospital night work interferes with junior doctors' ability to make the most of training opportunities in the community, where the pace of work is different and where other members of the health care team are even less understanding than non-medical hospital staff, when doctors arrive late or go to sleep on the job because of their nocturnal hospital activities. Community child health is a rapidly developing specialty with an urgent need to specialise more into such areas as neurodisability, social and behavioural problems, and population paediatrics. Ambulatory paediatrics and medical input to 'hospital at home' schemes (where the bulk of work is provided by paediatric community nurses) are other priority areas made more difficult to develop by staff being (over) involved with hospital inpatient work.

\section{(4) INABILITY TO MEET STANDARDS AND} GUIDELINES

Few small inpatient units can fulfil the Department of Health guidelines Welfare of Children and Young People in Hospital, ${ }^{3}$ nor all the recommendations of the Audit Commission report Children First ${ }^{1}$; Action for Sick Children (NAWCH) Charter recommendations ${ }^{4}$ and other professional advice ${ }^{5}$ are rarely fully complied with.

\section{(5) LIMITED CLINICAL AND INADEQUATE}

RESEARCH TRAINING EXPERIENCE

The low throughput in many units of serious and high dependency problems, the limited opportunities for 'shared care', and the rather numbing experience of rapid throughput low dependency problems, together (in inner cities especially) with a largely general practice clientele attending accident and emergency departments where cases are often seen by paediatric junior staff, all provide a threadbare experience for junior staff embarked on higher professional training and a less than thrilling experience for senior house officers (SHOs), especially with the present intolerable hours of work. The new post-Calman ${ }^{6}$ arrangements being introduced require a greatly shortened training period. Higher professional trainees will need a concentrated exposure to a wider range of clinical problems on a very structured basis, while the grinding present service load of SHOs will have to be lightened if general professional trainees in paediatrics are to have any hope of completing their part 2 membership in two or even three years in order to qualify for entry to higher professional training. We must ensure that the large majority of SHOs pass on to higher training in the minimal time if paediatrics is to compete with other disciplines. 'Senior' SHO posts, providing registrar level cover without full membership, should be strongly discouraged.

Research in paediatrics is at a low ebb. There are very few published articles from small paediatric inpatient units suggesting that, by this criterion at least, the research training needs of registrars and senior registrars are not being met. The half day or full days for research and study are often consumed in administration, correspondence, or emergency clinical cover for sick or absent colleagues. Few consultants in most small inpatient units find time to supervise, much less undertake, research even on clinical topics. The incomplete development of shared care, with most tertiary care still being provided in tertiary centres, means that junior staff there have reduced research opportunities as well and often complete an attachment without finishing any research project and with minimal or no publications.

A recent survey from North East Thames demonstrated major training deficiencies in 


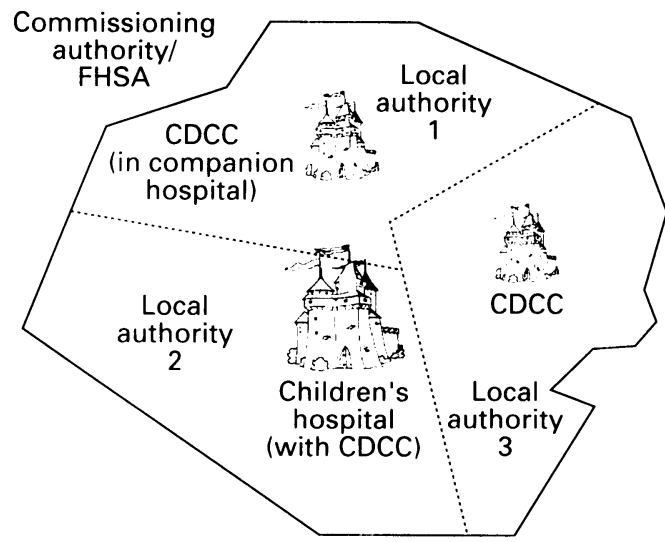

Comprehensive child health service comprising three CDCCs (one in the central children's hospital) and associated specialist community child health services.

'Special needs' care must be local authority boundary based to ensure effective integration with social services and education (20-50 000 children); population paediatrics relates to the commissioning authority/family health service authority/boundary (40-100 000 children).

most units ${ }^{7}$; registrars without part 2 membership of the Royal College of Physicians diploma had been appointed in over half the units; few junior doctors had protected or structured study and/or research time.

(6) STAFFING, HOURS OF WORK, AND QUALITY OF L.IFE

Junior doctors' hours are the key to change in the present situation. Many units are having trouble achieving 72 hours especially if no more than 56 hours are to be actually worked, even with four SHOs and four middle grade staff. Holiday cover and study leave, the latter increasingly, are difficult to arrange. Units undertaking neonatal intensive care need separate SHO rotas, and, if undertaking adequate throughput to maintain competence and cost efficiency, separate middle grade rotas as well. Partial or complete shifts are difficult

Table 1 A children's hospital

\section{Suggested components of a comprehensive children's unit/'children's hospital'}

1. Inpatients: Provision for $30-50+24$ hour beds. Two (perhaps more) ward areas would be needed, one with an infant orientation, the other with an adolescent area, both with adequate cubicles for infectious/immunocompromised cases

2. Day patients: Separate areas for surgery, day care/observation, and investigations $(-10$ spaces

3. Outpatients: Dedicated area for pacdiatrics, with sufficient space

4. Accident and emergency: Separate children's area, ideally adjacent to the paediatric outpatient/inpatient area, with interested 'ambulatory' paediatric consultant support

5. Intensive therapy unit (ITU): 2-4 paediatric spaces for single system problems - probably in association with adult ITU

6. Neonatal unit: 6-10 intensive care cots, together with appropriate 'special' and transitional care facilities, with 'high risk' obstetric service covering at least 10000 deliveries in association with surrounding midwifery only/GP units

7. Child mental health services, linked with community services

8. Child development centre: possibly off-site, but ideally adjacent to/part of paediatric outpatient area, with shared reception, waiting, play areas, and the like, to provide a local and, tient area, with shared reception, waiting, play areas, and the like,
probably, 'complex case' diagnostic service for surrounding areas

9. School room, teachers, play therapist $(s)$, play room

10. Parents' facilities

11. Paediatric orientated pathology, radiology, and support services

1. Paediatric orientated pathology, radiolog,

12. Paediatric social work: support on-site

14. Outreach community services: paediatric community nurses (at least six to cover the whole 10000 deliveries/year area) and associated consultant led medical staff, providing community secondary care, input to the surrounding CDCCs and child development/special needs work

15. Child health administration and computing to facilitate optimal integration between primary and secondary care services, for information exchange, to provide responsive back-up, support and training, and the organising of secondary (special needs) community care

16. Teaching, training areas

17. Office accommodation/support facilities, including a full time secretary/personal assistant for each consultant

18. Adjacent staff crêche (perhaps nursery) as 'normal child' training aid to arrange (and virtually impossible if community junior staff are to be included). Natural justice suggests that junior doctors should not be exempt from the likely universal introduction of a maximum 48 hour week. This will also apply to consultants. Attempts to substitute or complement SHOs or registrars in small units by using consultants and/or staff grades to provide on-call cover, will prove impossibly expensive and, if consultants are regularly expected to be resident (to undertake general practice accident and emergency work and obstetric assistance which could be provided by midwife practitioners), will further reduce the attractiveness of paediatrics as a specialty. Paediatrics is in crisis from many points of view, not least in the recruitment and retention of staff, particularly at consultant and higher training grade levels.

There are similar deficiencies in training opportunities, clinical experience and recruitment for children's trained nurses, children's therapists, and other staff in most present small paediatric units.

\section{Possible solutions}

(1) INCREASE MEDICAI. STAFF

This is unlikely to be cost effective but, more importantly, would further erode training opportunities and clinical competence in terms of experience with difficult problems. Increasing numbers of hospital staff would also further impede the necessary development of care for children, whenever possible, outside hospital.

(2) ESTABLISH A COMPREHENSIVE CHILDREN'S SERVICE WITH REORGANISATION OF INPATIENT BEDS

Groups of small inpatient units should come together, with secondary community child health services, to form a comprehensive children's service (figure). In general, these should be co-terminous with commissioning consortia/authority or family health service authority boundaries (serving a population of 40-100 000 children). One central site should be selected as a children's hospital - based within a district general hospital. This children's hospital would be a comprehensive children's unit (for components see table 1) and would provide sufficient inpatient throughput to maintain clinical competence, even with uncommon conditions and opportunities to specialise, including shared care with tertiary services; thus providing easily accessible, quality care, better training opportunities, and other benefits. There should be separate facilities for infants, isolation, older children and adolescents, as well as for education - a children's hospital school, with a critical mass of teachers to ensure coverage of the national curriculum, especially important for children with severe chronic or relapsing conditions who may otherwise have very deficient schooling.

Units without a 24 hour inpatient bed provision would operate as children's day care 
Table 2 CDCC

Components

1. Places/beds: $10 \pm$ for

Day case surgery (preoperative assessment and postoperative recovery)

Short term assessment/observation of cases which might require hospitalisation, fo example, for asthma

Day treatment, for example, chemotherapy for leukaemia/oncology shared care cases

2. Outpatient facilities: for

Local 'routine' referrals

Outreach clinics from tertiary centres or comprehensive children's unit

Urgent referrals from local general practitioners, community staff and (daytime) local accident and emergency service

3. Child development centre (CDC) for the local service, providing local assessments, some treatment facilities, and as a base for peripatetic special needs staff

Usually co-terminous with the local authority/local educational authority

Usually co-terminous with the local authority/local educational auth

Linked with the 'diagnostic' CDC at the related children's hospital
Shared reception, waiting, play areas, and staff if adjacent to the CDCC/children's

4. Staff: outpatient area, to ensure integration and efficiency

Consultant led, with regular, probably daily, consultant clinics. (Children's hospital, local community and visiting/other specialty consultants)

Junior paediatric/child health doctors might include staff grade for continuity, but mainly be senior/registrars/SHOs on rotational attachments who would continue to provide on-call/night time cover to the associated children's hospital

Local GPs might have short term clinical assistant attachments to complement consultant and perhaps other staff sessions in local general practitioner/primary care centres

Nursing (children's trained) and other staff might also rotate to the children's hospital to maintain confidence with paediatric advances and, perhaps, contribute to paediatric community nurse work in the local community

5. Continuing education for staff

Local as well as central sessions

Involve local primary health care team staff/GPs and

Others interested/involved with child health home services, and focused ambulatory provision in the local children's day care centre, should result in a better service than currently operates in many small isolated paediatric inpatient units. There are likely to be some difficulties with very isolated country units but even in this situation, most children requiring hospital inpatient care would likely be better in a hospital able to provide high dependency or specialist treatment. The argument is likely to turn on quality versus access. In most cases quality should win.

\section{(2) OTHER SERVICES}

(a) Obstetrics

The time is passing when paediatric inpatient care must be based on the perceived need to support obstetric services. Many women, supported by many midwives and the House of Commons Select Committee on Maternity Services, ${ }^{8}$ wish midwifery only units (associated with an increase in domicilary deliveries). There are clear recommendations. ${ }^{9}$ The BPA Health Services Committee Working Group document (Flexible Options for Paediatric Care) recommended that there be no paediatric cover out of hours for units that have fewer than 1500 births per year, ${ }^{10}$ but full resident experienced paediatric cover for larger units with more than 3000 births. For units between 1500 and 3000 deliveries, emergency neonatal care might be provided by neonatal nurse practitioners, midwives, general practitioners, anaesthetists, or obstetric medical staff. In general, high risk pregnancies should be managed in a large obstetric unit associated with a children's hospital which would have a neonatal intensive care unit and full paediatric support. munity child health service and with the children's hospital, often on rotation. It would provide an excellent training environment and fulfil most and more of the functions presently provided by most small paediatric units with inpatient beds.

\section{Possible problems with a comprehensive} children's service

(1) PUBLIC PERCEPTIONS ('DON'T CLOSE OUR PAEDIATRIC UNIT')

There is a widespread belief that there should be a fully equipped district general hospital on every street corner, able to deal with any emergency or medical problem. A major 'hearts and minds' education exercise will be required to persuade local people, general practitioners, community health councils, and local politicians that children requiring inpatient treatment would receive better and safer care in a properly staffed and properly structured unit even though this might necessitate the family travelling slightly further. Paediatric units without 24 hour inpatient provision would not be closed but would continue to provide most of the present services and more; only overnight stay would be affected for those few children needing it. Developments in community care, particularly paediatric community nursing/hospital at (c) Surgery

Surgeons operating on children in a unit without 24 hour inpatient provision would have to restrict their activities to day cases only. Any child unexpectedly requiring overnight care would be transferred to the children's hospital. 
This might result in a beneficial reduction in the number of occasional operators and occasional child anaesthetists.

\section{(3) MANAGEMENT AND MARKET PLACE} MEDICINE

The present unsatisfactory situation with paediatrics is not helped by the current plethora of competing trusts providing child health services, few on a cooperative, and fewer on a combined management, basis. The major trends in child health care over the twenty or thirty years before the recent NHS reforms were towards increasing cooperation between services for children, rather than competition. Most current central recommendations, for example, the recent Audit Commission report on community services for children, ${ }^{11}$ support cooperation and 'working together' and some purchasers see the benefits to children and families from collaborative working and integration between tertiary, secondary, and primary care.

A comprehensive children's service could be managed as a children's trust with service level agreements to the various host institutions providing children's hospital, day care centre, or other facilities. A comprehensive children's service would provide a truly child centred focus for care, would prove very attractive for staff development and training, and should be cost effective, as well as meeting the needs of children and families and the required standards and quality of care. Such an arrangement would provide an innovative model service applicable to other age and client groups.

\section{Conclusion}

Children's hospital departments and community child health units need to combine to form comprehensive specialist children's services; to meet changing child health needs, to provide better training and research opportunities, and to resolve the present crisis of morale and other difficulties facing current paediatric practice. The comprehensive children's service would comprise a children's hospital (within a district general hospital), extended secondary community child health services and CDCCs - mainly based in paediatric units presently providing 24 hour care. The comprehensive, specialist children's service should be managed on a combined basis, possibly as a children's trust and be closely integrated with local primary care and tertiary services. A reduction from the present number of paediatric inpatient units seems essential, to provide proper care of those children who really need inpatient care, to meet standards, quality, hours of work and training requirements, and to enable the necessary development of child and family focused, rather than hospital focused care.

1 Audit Commission. Children first: a study of hospital services. London: HMSO, 1993.

2 British Paediatric Association. The transfer of infants and children for surgery. (Report of the joint working group.) children for surgery.

3 Department of Health. Welfare of children and young people in hospital. London: HMSO, 1991.

4 National Association for the Welfare of Children in Hospital. The NAWCH charter. London: NAWCH, 1984

5 North East Thames Regional Paediatric Advisory Committee. Secondary health services for children and youn people. (Report of joint working party.) London: NE Thames Regional Health Authority, 1993.

6 Department of Health. Hospital doctors. Training for the future. (Report of the working group on specialis medical training.) London: Department of Health, 1993.

7 Pietroni M, Tinker J, West G. Paediatric training in north east Thames region. (Report of a regional survey 1993.) London: North East Thames Department of Postgraduate Medicine, British Postgraduate Medical ederation, 1994

8 Maternity services. (House of Commons health committe second report.) London: HMSO, 1992.

9 Department of Health. Changing childbirth. (Th Cumberlege report.) London: HMSO, 1993.

10 British Paediatric Association. Flexible options for paediatric care. (Report of a working group of the BPA health services committee.) London: BPA, 1993.

11 Audit Commission. Seen but not heard. Coordinating community child health and social services for children in need. London: HMSO, 1994. 